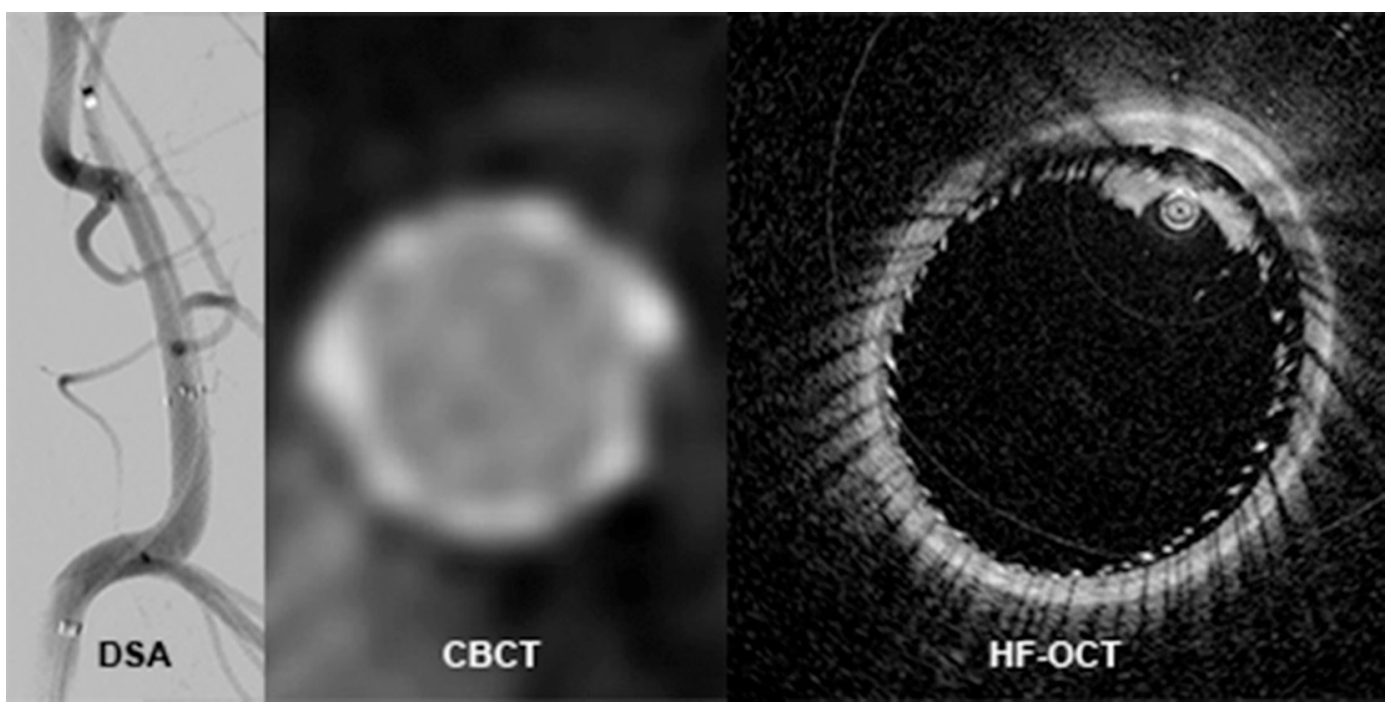

Abstract 0-008 Figure 1

clearing of highly attenuating erythrocytes for an optically clear field. In the pig study, the agreement by the reviewers (Fleiss kappa) regarding clot formation at 3 distinct locations along the flow diverter was $0.49,0.67$ and 0.90 for DSA, CBCT and HF-OCT, respectively. The agreement for diagnosing malapposition at these locations was $0.18,0.67$ and 0.87 for DSA, CBCT, and HF-OCT, respectively. In the porcine tortuosity model, in all cases, navigation and imaging was performed with uniform illumination and free from artifact.

Conclusion An HF-OCT device has been built that is compatible with neurointerventional surgery workflow and cerebrovascular anatomy. Preclinical data demonstrate consistent improvement to diagnose acute platelet aggregation and malapposition of flow diverters as compared to existing imaging modalities (figure 1).

Disclosures A. Puri: 1; C; NIH. M. Marosfoi: None. G. Ughi: 4; C; Gentuity. 5; C; Gentuity. R. King: None. E. Langan: None. J. Chueh: None. M. Gounis: 1; C; NIH.

\section{0-009 NEONATAL MRI FINDINGS ASSOCIATED WITH AGGRESSIVE EARLY CLINICAL COURSE IN NEWBORNS WITH VEIN OF GALEN MALFORMATION}

${ }^{1} \mathrm{~L}$ Arko IV*, ${ }^{2} \mathrm{D}$ Zurakowski, ${ }^{1} \mathrm{D}$ Obrach. ${ }^{1}$ Neurosurgery, Boston Children's Hospital, Boston, $M A_{;}{ }^{2}$ Anaesthesia, Boston Children's Hospital, Boston, MA

\subsection{6/neurintsurg-2019-SNIS.9}

Abstract Neonates born with vein of Galen malformations bifurcate into either (a) a cohort that experiences cardiopulmonary failure and/or early cerebral injury necessitating urgent neonatal embolization, or (b) a cohort that is clinically stable, in whom embolization can be safely deferred for months. There are no currently known imaging prognosticators that enable distinguishing these groups, and thus management is based solely on clinical factors, such as the Bicêtre criteria. In this study, MRI characteristics on DOL 1 images were evaluated in order to assess whether particular features correlated with the need for urgent neonatal intervention or neonatal death.

Methods Newborns with vein of Galen malformation who underwent DOL 1 MRI scans from 2007-2018 at a single tertiary referral center were evaluated. 18 anatomic vessel parameters were measured and statistically compared to ascertain whether any parameters correlated with neonatal death or urgent neonatal embolization (neonatal at-risk cohort, NAR) versus survival to treatment in infancy (infantile treatment cohort, IT). Vessels assessed included the median prosencephalic varix, straight/falcine sinus, sigmoid sinuses, internal jugular veins, internal carotid arteries, and basilar artery. Continuous variables were compared using the nonparametric Mann-Whitney U test. Categorical data were compared using chi-square or Fisher's exact test. Multivariable logistic regression test was applied to identify independent predictors that together can best differentiate the NAR and IT cohorts with area under the curve (AUC) used to determine predictive accuracy.

Results There were 21 patients in the neonatal at-risk cohort, and 11 patients in the infantile treatment cohort. Of the 18 variables tested, 12 were found to be significant, including: diameter and circumference of the straight/falcine sinus at its narrowest point, basilar artery diameter, internal carotid artery diameters, sigmoid sinus circumferences, and internal jugular vein diameters. Interestingly, neither the caliber (diameters/volume) nor the shape of the prosencephalic varix itself was found to distinguish the two cohorts. An occipital sinus was visualized for all patients in the NAR cohort versus $45 \%$ of the IT cohort $(\mathrm{P}<0.001)$. Starting with five significant variables by univariate analysis, multivariable regression analysis confirmed that straight/falcine sinus caliber at its narrowest, combined with large RICA diameter, provided the most predictive model (AUC $=0.870,95 \% \mathrm{CI}: 0.746-0.994, \mathrm{P}<0.001$ ).

Conclusion Based on 32 newborns with vein of Galen malformation, MRI measurements on DOL 1 were significantly different between patients at risk of neonatal death or need for urgent embolization, compared to patients in whom treatment was safely deferred until infancy. As these cohorts differ significantly in overall risk of mortality and severe neurodevelopmental morbidity, this early prognostication can be of great potential help to both families and caregivers. These findings require validation on a larger newborn population to provide evidence of generalizability.

Disclosures L. Arko: None. D. Zurakowski: None. D. Obrach: None. 